\title{
The Role of Query Sessions in Interpreting Compound Noun Phrases
}

\author{
Marius Paşca \\ Google Inc. \\ 1600 Amphitheatre Parkway \\ Mountain View, California 94043 \\ mars@google.com
}

\begin{abstract}
The meaning of compound noun phrases can be approximated in the form of lexical interpretations extracted from text. The interpretations hint at the role that modifiers play relative to heads within the noun phrases. In a study examining the role of query sessions in explaining compound noun phrases, candidate interpretations of compound noun phrases are extracted from pairs of queries that belong to the same query session. Experimental results over multiple evaluation sets of noun phrases show a higher accuracy of the interpretations when extracted from query sessions rather than from individual queries.
\end{abstract}

\section{Categories and Subject Descriptors}

I.2.7 [Artificial Intelligence]: Natural Language Processing; H.3.1 [Information Storage and Retrieval]: Content Analysis and Indexing; H.3.3 [Information Storage and Retrieval]: Information Search and Retrieval; I.2.6 [Artificial Intelligence]: Learning

\section{General Terms}

Algorithms, Experimentation

\section{Keywords}

Compound noun phrases, compositional concepts, open-domain information extraction, knowledge acquisition

\section{INTRODUCTION}

Motivation: A necessary step towards understanding the meaning of compound noun phrases (e.g., "beatles songs", "french macarons", "ayurvedic medicinal plants") is to understand the roles that modifiers (beatles, french, ayurvedic) play within the noun phrases. Often, the roles can be approximated lexically, in the form of string interpretations ("composed by", "from", "used in") that serve as connectors within what are effectively paraphrases ("songs composed by beatles", "macarons from france", "medicinal plants used in ayurveda") of the compound noun phrases.

Evidence available within, and collected from, large text collections should be helpful in the analysis of compound noun phrases, Permission to make digital or hard copies of all or part of this work for personal or classroom use is granted without fee provided that copies are not made or distributed for profit or commercial advantage and that copies bear this notice and the full citation on the first page. Copyrights for components of this work owned by others than the author(s) must be honored. Abstracting with credit is permitted. To copy otherwise, or republish, to post on servers or to redistribute to lists, requires prior specific permission and/or a fee. Request permissions from Permissions@ acm.org. CIKM'15, October 19-23, 2015, Melbourne, VIC, Australia

Copyright is held by the owner/author(s). Publication rights licensed to ACM. ACM 978-1-4503-3794-6/15/10\$15.00

DOI: http://dx.doi.org/10.1145/2806416.2806571 and allow for the extraction of relevant interpretations. Indeed, textual document collections are a useful source for extracting paraphrases of compound noun phrases $[19,36]$. As an alternative to using document collections, collections of Web search queries produce interpretations that may be more accurate [28] than when extracted from documents, even if the source queries are available simply as a set of queries that are independent from one another. Contributions: Expanding upon [28], the experiments reported in this paper examine the role of Web search queries vs. query sessions in uncovering the semantics of open-domain class labels in particular; and of compound noun phrases in general. Queries and query sessions are the source for candidate lexical interpretations of compound noun phrases. The interpretations turn implicit properties or subsuming roles ("composed by", "from", "used in") that modifiers (beatles, french, ayurvedic) play within longer noun phrases ("beatles songs", "french macarons", "ayurvedic medicinal plants") into concrete strings. The roles of modifiers relative to heads of noun phrase compounds cannot be characterized in terms of a finite list of possible compounding relationships [10]. Hence, the interpretations are not restricted to a closed, pre-defined set. In experiments comparing query sessions and independent queries, over evaluation sets of noun phrases from multiple sources, more accurate interpretations are extracted from query sessions.

\section{INTERPRETING NOUN PHRASES}

Compounds: Let $N$ be a compound noun phrase, containing a head $H$ preceded by modifiers $M$. Each of $H$ and $M$ may contain one or multiple tokens. Being a compound, the sequence of modifiers and head in $N$ act as a single noun [10,16], which refers to a concept whose meaning is based on the semantics of its components $[24,35]$.

Hypothesis 1: If $N$ is relevant and of interest to Web users, then in a sufficiently large corpus it will eventually be referred to in relatively more verbose search queries, which reveal the implicit role that modifiers $M$ play relative to the head $H$.

Hypothesis 2: If $N$ is relevant and of interest to Web users, then in a sufficiently large corpus it will eventually be referred to in the same query session [34] as more verbose search queries, which reveal the implicit role that modifiers $M$ play relative to the head $H$. Extraction from Queries: Figure 1 gives an overview of the role of queries and query sessions in extracting interpretations of compound noun phrases. The compound noun phrases to be interpreted are available as an input vocabulary of noun phrases. For example, the vocabulary might include the noun phrases "beatles songs" and "ayurvedic medicinal plants". The source of candidate interpretations takes the form of anonymized queries, from which possible interpretations of the noun phrases must be extracted. The queries are available as a set of pairs of anonymized queries submitted 


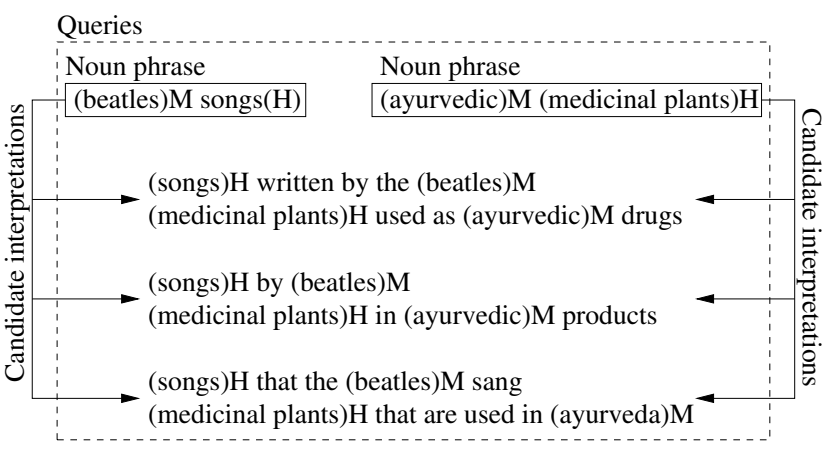

Figure 1: Extraction of interpretations of noun phrases from queries ( $\mathrm{H}, \mathrm{M}=$ head and modifier)

within the same query sessions, and also as a set of independent anonymized queries, collected from the queries that form the pairs. For example, the set of queries may include "songs written by the beatles" and "medicinal plants in ayurvedic products". Optionally, if the queries are available as query sessions rather than merely as a set of queries, the queries "beatles songs" and "songs written by the beatles", or "ayurvedic medicinal plants" and "medicinal plants in ayurvedic products", may be pairs that belong to the same query sessions.

The extraction consists of several steps [28]: (1) the selection of a subset of queries that may be candidate interpretations of some yet-to-be-specified noun phrases; (2) the matching of the selected queries to the noun phrases to interpret; and (3) the aggregation of matched queries into candidate interpretations extracted for a noun phrase.

Selecting Candidate Interpretations: As a pre-requisite, the noun phrases to interpret and queries are both part-of-speech tagged [5]. A query is deemed to be a candidate interpretation of a hypothetical compound noun phrase that might consist in a modifier $M$ immediately followed by a head $H$ (e.g., "(ayurvedic) ${ }_{M}$ (medicinal plants $)_{H}$ "), if the query matches one of the following patterns [28]:

- passive constructs, for example "(medicinal plants $)_{H}$ used as (ayurvedic) ${ }_{M}$ drugs";

- prepositional constructs, for example "(medicinal plants $)_{H}$ in (ayurvedic) ( products";

- relative pronoun constructs, e.g., "(medicinal plants $)_{H}$ that are used in (ayurveda) ${ }_{M}$ ".

The patterns effectively split matching queries into four consecutive sequences of tokens $Q=\left[\begin{array}{llll}Q_{1} & Q_{2} & Q_{3} & Q_{4}\end{array}\right]$, where $H$ and $M$ correspond to $Q_{1}$ and $Q_{3}$, and $Q_{4}$ may be empty. For example, in the lower portion of Figure 1, one of the patterns matches the query "(medicinal plants $)_{H}$ in (ayurvedic) ${ }_{M}$ products", thus splitting the query into "medicinal plants" as $Q_{1}$, "in" as $Q_{2}$, "ayurvedic" as $Q_{3}$ and "products" as $Q_{4}$.

Mapping Noun Phrases to Interpretations: Each noun phrase to interpret is split into all possible decompositions of two consecutive sequences of tokens $N=\left[N_{1} N_{2}\right]$, where the two sequences correspond to a hypothetical modifier and a hypothetical head of the noun phrase. For example, the noun phrase "ayurvedic medicinal plants" is split into ["ayurvedic medicinal", "plants"] and separately into ["ayurvedic", "medicinal plants"]. If $N_{1}$ and $Q_{3}$, and $N_{2}$ and $Q_{1}$ respectively, match, then the matching query $Q$ (e.g., "(medicinal plants $)_{H}$ in (ayurvedic) ${ }_{M}$ products") is retained as a candidate interpretation of the noun phrase $N$ ("(ayurvedic) ${ }_{M}$ (medicinal plants $)_{H}$ ”), as shown in the middle portion of Figure 1. If the noun phrases are interpreted using query sessions instead of independent queries, the matching query $Q$ and the noun phrase $N$ must satisfy an additional constraint, namely to be one of the pairs of queries that are available as part of the same query session. Otherwise, the matching query $Q$ is discarded.

Mapping via Modifier Variants: At its simplest, the matching of the hypothetical modifier relies on strict string matching. Alternatively, original modifiers in the noun phrases to interpret may be matched to queries via expansion variants. Variants are phrases that likely play the same role, and therefore share interpretations, as modifiers relative to the head in a noun phrase. Variants allow for the extraction of candidate interpretations that may otherwise not be available in the input data. For example, the variant rolling stones available for beatles allows for the matching of beatles in

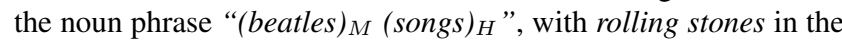
query "( songs $)_{H}$ played by (rolling stones $)_{M}$ ". The candidate interpretation " $(\text { songs })_{H}$ played by (beatles $)_{M}$ " is extracted for the noun phrase "(beatles $)_{M}$ (songs) $)_{H}$ ", even though the query "songs played by beatles" might not be present among the input queries.

Possible sources of variants include, among others, distributionally similar phrases [21], where the phrases most similar to a modifier would act as its variants. Mappings from adjectival modifiers in noun phrases (e.g., ayurvedic in "ayurvedic medicinal plants" in Figure 1) into the nominal counterparts (e.g., ayurveda) that are likely to occur in interpretations (e.g., "(medicinal plants $)_{H}$ that are used in (ayurveda) ${ }_{M}$ ”) are also useful. Concretely, as described later in the experimental setting, variants are generated using WordNet [12], distributional similarities and Wikipedia [33]. Aggregation of Candidate Interpretations: Candidate interpretations of a noun phrase are aggregated from source queries that matched the noun phrase. The frequency score of a candidate interpretation is the weighted sum of the frequencies of source queries from which the candidate interpretation is collected, possibly via variants of modifiers. In the weighted sum, the weights are similarity scores between the original modifier from the noun phrase, on one hand, and the variant from the source query into which the modifier was mapped, on the other hand [28]. The weights for the variants beatles and rolling stones relative to the original modifier beatles are 1.0 (identity) and within [0.0, 1.0] (distributional similarity), whereas the weights of adjectival modifiers such as ayurveda for ayurvedic are 1.0. Separately from the frequency score, a penalty score is computed that penalizes interpretations containing extraneous tokens. Specifically, the penalty counts the number of nouns or adjectives located outside the modifier and head. For example, the penalty scores assigned to the interpretations "(songs $)_{H}$ by $(\text { beatles })_{M}$ " and "(medicinal plants $)_{H}$ that are used in (ayurveda) ${ }_{M}$ " are 0 . In comparison, the penalty scores for the interpretations "(songs $)_{H}$ that (beatles $)_{M}$ play" and "(medicinal plants $)_{H}$ in (ayurvedic) $)_{M}$ products” are 1 . From among candidate interpretations extracted for a noun phrase, interpretations whose penalty score is higher than 1 are discarded. Candidate interpretations extracted for a noun phrase are ranked in increasing order of their penalty scores or, in case of ties, in decreasing order of their frequency scores.

\section{EXPERIMENTAL SETTING}

Sources of Textual Data: The experiments rely on a random sample $\mathbf{S}$ of around 3 billion unique pairs of fully-anonymized Web search queries in English. The sample is drawn from pairs of queries within the same query session(s), as submitted to a general-purpose Web search engine. A session contains queries submitted in relatively quick succession by the same anonymized user [34]. The queries from the query pairs in the sample $S$ are separately organized as a sample $\mathbf{Q}$ of around 1.5 billion unique queries. Each 


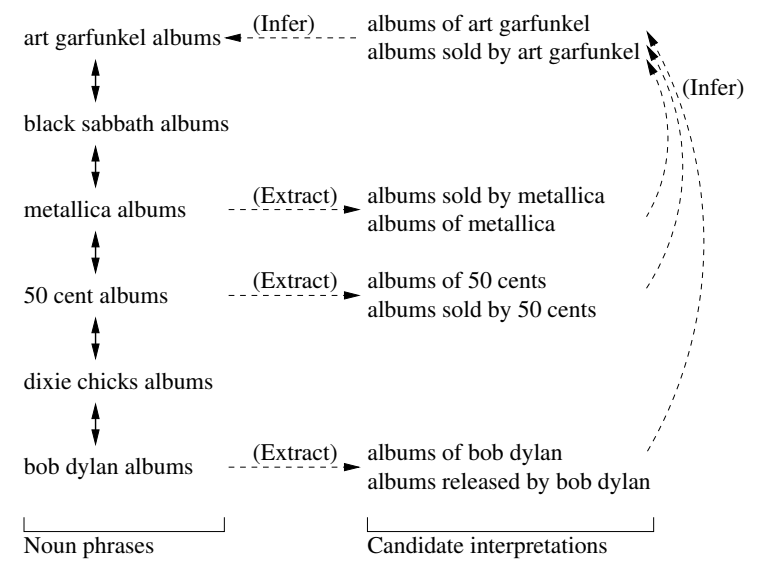

Figure 2: Acquisition of interpretations of compound noun phrases via wiki-templ variants

query in Q is available independently from other queries, and is accompanied by its frequency of occurrence in the query logs. In this setup, each query in Q also appears as a query in some query pair in $\mathrm{S}$. Conversely, each query in a query pair in $\mathrm{S}$ also appears as a query in Q.

Experimental Runs: The experiments consist in extracting interpretations from the independent queries $\mathrm{Q}$, in the case of run $\mathbf{R}_{Q}$, as introduced in [28]; or from the query sessions $\mathrm{S}$, in the case of run $\mathbf{R}_{S}$, as proposed here.

Sources of Variants: The original form of the modifiers is denoted as orig-phrase. Three types of variant phrases are collected for the purpose of matching modifiers within noun phrases to interpret, with phrases from queries. Relations encoded as ValueOf, Related-Noun and Derivationally-Related relations in WordNet [12] are the source of adj-noun variants. They map around 6,000 adjectives into one or more nouns (e.g., (french $\rightarrow$ france), (electric $\rightarrow$ electricity), (aquatic $\rightarrow$ water $)$ ). A repository of distributionally similar phrases, collected in advance [21,29] from a sample of around 200 million Web documents in English, is the source of dist-sim variants. For each of around 1 million phrases, the variants consist of their 50 most similar phrases (e.g., art garfunkel $\rightarrow$ $\{$ carly simon, melissa manchester, aaron neville, .. \}). A snapshot of all Wikipedia [33] articles in English, as available in June 2014, is the source of wiki-templ variants. For each of around 50,000 phrases, their wiki-templ variants are collected from Wikipedia categories sharing a common parent Wikipedia category (e.g., "albums by artist") and having a common head ("art garfunkel albums", "black sabbath albums", "metallica albums"). The different modifiers (art garfunkel, black sabbath, metallica) that accompany the shared head are collected as variants of one another. Among the four types of variants, wiki-templ variants are applied only when the noun phrase to interpret, and the source Wikipedia category names from which the variants were collected, have the same head. For example, $X=$ art garfunkel $\rightarrow$ \{ black sabbath, metal lica, 50 cent,..$\}$ is applied only in the context of the noun phrase "X albums". As shown in Figure 2, the extraction of interpretations via some of the variants allows for effectively inferring interpretations via other variants, as long as the noun phrases share the same head. When computing the frequency score of a candidate interpretation as the weighted sum of the frequencies of source queries, the weights assigned are 1.0, for orig-phrase, adj-noun and wiki-templ variants; and the available distributional similarity scores within $[0.0,1.0]$, for dist-sim variants.
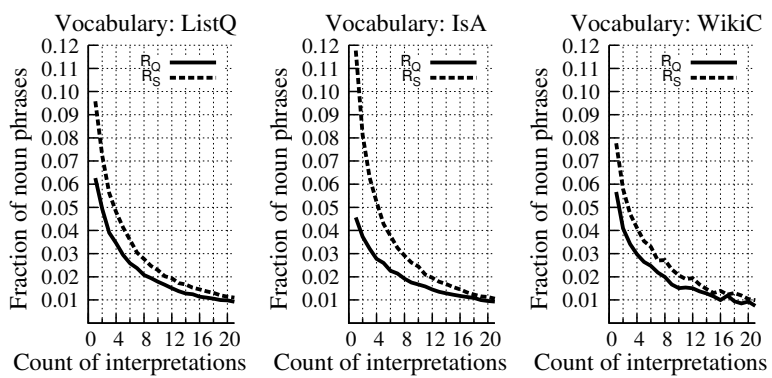

Figure 3: Distribution of the number of interpretations extracted per noun phrase, over noun phrases with some extracted interpretation(s). Computed over various vocabularies of noun phrases

Vocabularies of Noun Phrases: The experiments acquire interpretations from queries, for noun phrases from three vocabularies. ListQ is a set of phrases $X$ (e.g., "aramaic words") from queries in the form [list of $X]$, where the frequency of the query $[X]$ is at most 100 times higher than the frequency of the query [list of $X$ ], and the frequency of the latter is at least 5. IsA is a set of class labels (e.g., "academy award nominees"), originally extracted from Web documents via Hearst patterns [15], and associated with at least 25 instances each (e.g., zero dark thirty). WikiC is a set of Wikipedia categories that contain some tokens in lowercase beyond prepositions and determiners, and whose heads are plural-form nouns (e.g., "french fiction writers"). Only phrases that are one of the fulllength queries from the input set of Web search queries are retained in the respective sets, as vocabularies of noun phrases to interpret; other phrases are discarded.

\section{EVALUATION RESULTS}

Relative Coverage: Because it is not feasible to manually compile the exhaustive sets of all string forms of valid interpretations of all (or many) noun phrases, we compute relative instead of absolute coverage. At least one interpretation is extracted from query sessions and independent queries for more than 500,000 of the noun phrases from all input vocabularies. Considering only that subset, Figure 3 shows the distribution of the number of candidate interpretations extracted per noun phrase. Given a count of extracted candidate interpretations fixed between 1 and 20, run $\mathrm{R}_{S}$ consistently extracts that number of interpretations for a higher fraction of the noun phrases from the input vocabularies than $\mathrm{R}_{Q}$ does. The apparent advantage of run $\mathrm{R}_{S}$ over $\mathrm{R}_{Q}$ in fact reflects its inability to extract as many candidate interpretations as $\mathrm{R}_{Q}$ does. Indeed, although not shown in the graphs, more than 100 interpretations are extracted per noun phrase for $14.5 \%\left(\mathrm{R}_{Q}\right)$ vs. $5.5 \%\left(\mathrm{R}_{S}\right)$ of the noun phrases in ListQ, $24.8 \%\left(\mathrm{R}_{Q}\right)$ vs. $5.6 \%\left(\mathrm{R}_{S}\right)$ in IsA, and $16.7 \%\left(\mathrm{R}_{Q}\right)$ vs. $7.1 \%\left(\mathrm{R}_{S}\right)$ in WikiC.

Precision of Interpretations: From an input vocabulary, an initial weighted sample of 150 noun phrases with some interpretations extracted in both runs $\mathbf{R}_{Q}$ and $\mathbf{R}_{S}$ is manually inspected. The sampling weight is the frequency of the noun phrases as queries. A noun phrase from the selected sample is either retained, or discarded if deemed to be a non-interpretable phrase. A noun phrase is not interpretable if it is in fact an instance ("new york", "alicia keys") rather than a class; or it is not a properly formed noun phrase ("watch movies"); or does not refer to a meaningful class ("3 significant figures"). The manual inspection ends, once a sample of 100 noun phrases has been retained. The procedure gives weighted random samples of 100 noun phrases, drawn from each 
\begin{tabular}{|l|l|}
\hline Eval Set & Sample of Noun Phrases \\
\hline
\end{tabular}

\begin{tabular}{|r|l|}
\hline \hline ListQ & $\begin{array}{l}\text { ayurvedic medicinal plants, black history people, } \\
\text { good short stories, health care reform issues, hypoal- } \\
\text { lergenic makeup brands, jive songs, male celebrities, } \\
\text { periodic functions, plumbing snakes, simple carbs }\end{array}$ \\
\hline IsA & $\begin{array}{l}\text { automotive parts, casual shirts, classic rock bands, lit- } \\
\text { erature review, louis vuitton outlet, pc solutions, pdf } \\
\text { reader, private equity firms, unix command, word- } \\
\text { press plugins }\end{array}$ \\
\hline WikiC & $\begin{array}{l}\text { carnivorous plants, differentiation rules, free online } \\
\text { games, language tests, online games, time zones, toy } \\
\text { dogs, viz characters, wedding dresses, wii accessories }\end{array}$ \\
\hline
\end{tabular}

Table 1: Samples from evaluation sets of noun phrases in each vocabulary

\begin{tabular}{|c|c|}
\hline \multicolumn{2}{|c|}{ Examples of Correctness Annotations } \\
\hline Noun Phrase & Interpretation \\
\hline \multicolumn{2}{|c|}{ Correctness label: correct generic (numeric value: 1.0 ): } \\
\hline final cut pro 7 shortcuts & shortcuts of final cut pro 7 \\
\hline military ranks & ranks in the military \\
\hline pain assessment tool & assessment tool of pain \\
\hline stephen king book & book by stephen king \\
\hline \multicolumn{2}{|c|}{ Correctness label: correct specific (numeric value: 1.0 ): } \\
\hline android tablet & tablet with android os \\
\hline ayurvedic medicinal plants & medicinal plants used in ayurveda \\
\hline flash games & games made with flash \\
\hline omega 3 foods & foods that provide omega 3 \\
\hline \multicolumn{2}{|c|}{ Correctness label: okay (numeric value: 0.5 ): } \\
\hline green technology & technology used for green building \\
\hline lenovo laptops & laptops of lenovo company \\
\hline nursery rhymes & rhymes for kids in nursery \\
\hline toy dogs & dogs that are toy size \\
\hline \multicolumn{2}{|c|}{ Correctness label: incorrect (numeric value: 0.0 ): } \\
\hline cartoon characters & characters in cartoon network \\
\hline flash games & games designed by flash \\
\hline mexican films & films about mexican culture \\
\hline omega watches & watches that look like omega \\
\hline
\end{tabular}

Table 2: Examples of interpretations manually annotated with each correctness label

of the ListQ, IsA and WikiC vocabularies. The samples, shown in Table 1, constitute the evaluation sets of phrases ListQ, IsA and WikiC, over which precision of interpretations is computed.

Following evaluation methodology introduced for other opendomain information extraction tasks, the top 20 interpretations extracted in an experimental run for each evaluation phrase are manually annotated with correctness labels. As shown in Table 2, an interpretation is annotated as: correct and generic, or correct and specific, if relevant; okay, if useful but containing non-essential information; or wrong. To compute the precision score over an evaluation set of phrases, the correctness labels are converted to numeric values. Precision of a ranked list of extracted interpretations is the average of the correctness values of the interpretations in the list.

Table 3 provides a comparison of precision scores at various ranks in the extracted lists of interpretations, as an average over all phrases from an evaluation set. Several conclusions can be drawn from the results. First, at a given rank, the average precision of a run varies from one evaluation set to another, since the evaluation sets are selected from vocabularies from different sources. Second, precision levels are encouraging for all runs. Third, with the exception of precision at rank 1 for the IsA evaluation set, run $\mathrm{R}_{S}$ produces more accurate interpretations than $\mathrm{R}_{Q}$ across all ranks and across all evaluation sets. In particular, when considering the top 20 interpretations extracted for all phrases from the union of all evaluation

\begin{tabular}{|r|l|c|c|c|c|c|}
\hline $\begin{array}{c}\text { Eval } \\
\text { Set }\end{array}$ & \multirow{2}{*}{ Run } & \multicolumn{5}{|c|}{ Precision @N } \\
\cline { 3 - 7 } & & $@ 1$ & $@ 3$ & 05 & $@ 10$ & $@ 20$ \\
\hline \hline ListQ & $\mathrm{R}_{Q}$ & 0.824 & 0.742 & 0.656 & 0.541 & 0.443 \\
\cline { 2 - 7 } & $\mathrm{R}_{S}$ & 0.886 & 0.795 & 0.726 & 0.639 & 0.549 \\
\hline IsA & $\mathrm{R}_{Q}$ & 0.790 & 0.636 & 0.533 & 0.429 & 0.336 \\
\cline { 2 - 7 } & $\mathrm{R}_{S}$ & 0.772 & 0.691 & 0.631 & 0.541 & 0.443 \\
\hline \multirow{2}{*}{ WikiC } & $\mathrm{R}_{Q}$ & 0.747 & 0.649 & 0.586 & 0.448 & 0.336 \\
\cline { 2 - 7 } & $\mathrm{R}_{S}$ & 0.801 & 0.717 & 0.662 & 0.568 & 0.478 \\
\hline Union & $\mathrm{R}_{Q}$ & 0.787 & 0.676 & 0.592 & 0.473 & 0.372 \\
\cline { 2 - 7 } of all & $\mathrm{R}_{S}$ & 0.820 & 0.734 & 0.673 & 0.583 & 0.490 \\
\cline { 2 - 7 } & + vs.R & $\mathbf{+ 4 . 2 \%}$ & $\mathbf{+ 8 . 6 \%}$ & $\mathbf{+ 1 3 . 7 \%}$ & $\mathbf{+ 2 3 . 3 \%}$ & $\mathbf{+ 3 1 . 7 \%}$ \\
\hline
\end{tabular}

Table 3: Average precision, at various ranks in the ranked lists of interpretations extracted for noun phrases from various sets of evaluation phrases. Computed for each run, as an average over each evaluation set, and as an average over the union of all evaluation sets

Run: Eval Phrase (P@ $@) \rightarrow$ [Ranked Candidate Interpretations] $\mathrm{R}_{Q}:$ atm card $(0.00) \rightarrow$ [card stuck in atm, card retained by atm, card retained in atm, card captured by atm, card eaten by atm,...] $\mathrm{R}_{S}^{-}: \mathrm{atm}^{-}$card $(1.00)^{-} \rightarrow$ [card used in atm, card for atm]

$\mathrm{R}_{Q}:$ ayurvedic medicinal plants $(1.00) \rightarrow$ [medicinal plants in ayurveda, medicinal plants used in ayurveda, medicinal plants of ayurveda, medicinal plants used by ayurveda, medicinal plants used in ayurvedic medicines, ..]

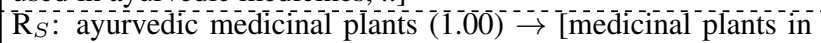
ayurveda, medicinal plants used in ayurveda, medicinal plants of ayurveda, medicinal plants used by ayurveda, medicinal plants used in ayurvedic medicines, ...

$\mathrm{R}_{Q}$ : chemical processes $(0.40) \rightarrow$ [processes of chemical weathering, processes in chemistry, processes involved in chemical weathering, processes of chemistry, processes in chemical weathering, ..]

$\mathrm{R}_{S}^{-}$: chemical processes $(1.00) \stackrel{-}{\rightarrow}$ [processes in chemistry, processes of chemistry, processes that involve chemistry, processes involved in chemistry, processes related to chemistry, ..]

$\mathrm{R}_{Q}:$ michael jackson song $(0.90) \rightarrow$ [song by michael jackson, song of michael jackson, song from michael jackson, song sung by michael jackson, song written by michael jackson, ...]

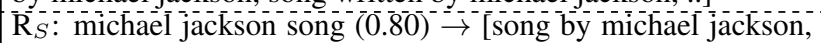
song of michael jackson, song from michael jackson, song sung by michael jackson, song for michael jackson, ..]

$\mathrm{R}_{Q}:$ special diet $(0.10) \rightarrow$ [diet with special $\mathrm{k}$, diet of special $\mathrm{k}$, diet for special forces, diet for special needs, diet before a special event, ...]

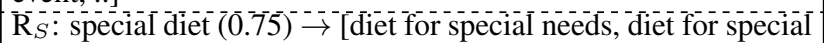
conditions, ..]

$\mathrm{R}_{Q}$ : uk record companies $(0.60) \rightarrow$ [record companies in the uk, record companies in uk, record companies of uk, record companies in london uk, ...]

$\mathrm{R}_{S}$ : uk record companies $(0 . \overline{7} 5) \rightarrow$ [record companies in the uk, record companies in uk, record companies of uk, record companies in london uk]

Table 4: Top candidate interpretations extracted in the experimental runs for a sample of noun phrases from the ListQ evaluation set $(P @ 5=$ precision at rank 5 in the ranked list of extracted interpretations)

sets, about half of the interpretations are correct, for $\mathrm{R}_{S}$; vs. slightly over a third, for $\mathrm{R}_{Q}$. At rank 1 , the precision of $\mathrm{R}_{S}$ is higher than 0.8 . Within an evaluation set, the accuracy of extracted interpretations varies between the experimental runs, as well as from one evaluation phrase to another, as illustrated in Table 4.

Presence of Relevant Interpretations: Since sometimes it is difficult to even manually enumerate as many as 20 distinct, relevant 


\begin{tabular}{|c|c|c|c|c|c|c|}
\hline \multirow[t]{2}{*}{ Eval Set } & \multirow[t]{2}{*}{ Run } & \multicolumn{5}{|c|}{$\begin{array}{l}\text { Average presence of any interpreta- } \\
\text { tions annotated as correct }\end{array}$} \\
\hline & & @ 1 & @3 & $@ 5$ & $@ 10$ & $@ 20$ \\
\hline \multicolumn{7}{|c|}{ Correctness label: correct generic, or correct specific: } \\
\hline \multirow[t]{2}{*}{ ListQ } & $\mathrm{R}_{Q}$ & 0.795 & 0.875 & 0.898 & 0.920 & 0.932 \\
\hline & $\mathbf{R}_{S}$ & 0.852 & 0.909 & 0.920 & 0.932 & 0.943 \\
\hline \multirow[t]{2}{*}{ IsA } & $\mathrm{R}_{Q}$ & 0.765 & 0.852 & 0.852 & 0.876 & 0.901 \\
\hline & $\mathbf{R}_{S}$ & 0.753 & 0.864 & 0.876 & 0.889 & 0.889 \\
\hline \multirow[t]{2}{*}{ WikiC } & $\mathrm{R}_{Q}$ & 0.711 & 0.831 & 0.904 & 0.940 & 0.940 \\
\hline & $\mathbf{R}_{S}$ & 0.771 & 0.879 & 0.940 & 0.964 & 0.964 \\
\hline \multicolumn{7}{|c|}{ Correctness label: correct specific only: } \\
\hline \multirow[t]{2}{*}{ ListQ } & $\mathrm{R}_{Q}$ & 0.034 & 0.284 & 0.386 & 0.545 & 0.614 \\
\hline & $\mathrm{R}_{S}$ & 0.068 & 0.307 & 0.420 & 0.568 & 0.614 \\
\hline \multirow[t]{2}{*}{ IsA } & $\mathrm{R}_{Q}$ & 0.086 & 0.210 & 0.309 & 0.506 & 0.593 \\
\hline & $\mathbf{R}_{S}$ & 0.086 & 0.247 & 0.346 & 0.531 & 0.568 \\
\hline \multirow[t]{2}{*}{ WikiC } & $\mathrm{R}_{Q}$ & 0.084 & 0.217 & 0.398 & 0.530 & 0.639 \\
\hline & $\mathbf{R}_{S}$ & 0.133 & 0.241 & 0.410 & 0.590 & 0.651 \\
\hline
\end{tabular}

Table 5: Average of scores indicating the presence or absence of any interpretations annotated as correct. Computed over interpretations extracted up to various ranks in the ranked lists of extracted interpretations

string forms of interpretations of a given noun phrase, measuring precision at a particular rank (e.g., 20) in a ranked list of interpretations may be too conservative. Table 5 summarizes a different type of scoring metric, namely the presence of any relevant interpretation, among the interpretations extracted up to a particular rank. Relevance is flexibly defined, by requiring the interpretations to have been assigned a certain correctness label, then computing the average number of evaluation phrases for which such interpretations are present up to a particular rank. Intuitively, the resulting scores at a given rank may be lower for $\mathrm{R}_{S}$ than for $\mathrm{R}_{Q}$. Indeed, as $\mathrm{R}_{S}$ effectively attempts to discard incorrect interpretations that $\mathrm{R}_{Q}$ would otherwise produce, it may occasionally discard relevant interpretations. Especially when few interpretations are extracted in the first place, discarding relevant interpretations may adversely affect the scores measuring the presence of any relevant interpretations. However, even as they are affected by this phenomenon, the scores in Table 5 are still competitive for $\mathrm{R}_{S}$ relative to $\mathrm{R}_{Q}$. The scores indicate that at least one of the top 5 interpretations is correct and specific for about 1 out of 3 noun phrases in the evaluation sets on average.

Popular Heads, Modifiers and Interpretations: The most frequent heads induced by interpretations extracted in $\mathrm{R}_{S}$ are shown in Figure 4. Although the incorrect head wikipedia is present in the upper graph, most of the heads and modifiers seem plausible, suggesting that the input vocabulary does contain reasonably clean compound noun phrases. In the lower graph, the prominence of location-related phrases (e.g., $u k$, indian) among the most popular modifiers for the ListQ vocabulary is not unexpected, but is not characteristic for all vocabularies. For example, in the case of the IsA vocabulary, the most popular modifiers are business, social, good, human, famous, with the earliest location-related modifier american being only the 24th most popular modifier.

Figure 5 illustrates the most popular interpretations extracted for noun phrases in the ListQ vocabulary. For example, if interpretations are restricted to those containing more than just prepositions and determiners, the ninth most popular interpretation is " $(H)$ written by $(M)$ ", where $\mathrm{H}$ and $\mathrm{M}$ stand for the induced heads and modifiers respectively.

Impact of Variants: Variants of modifiers provide alternatives in identifying and extracting candidate interpretations, even when the modifiers from the noun phrases are not present in their original

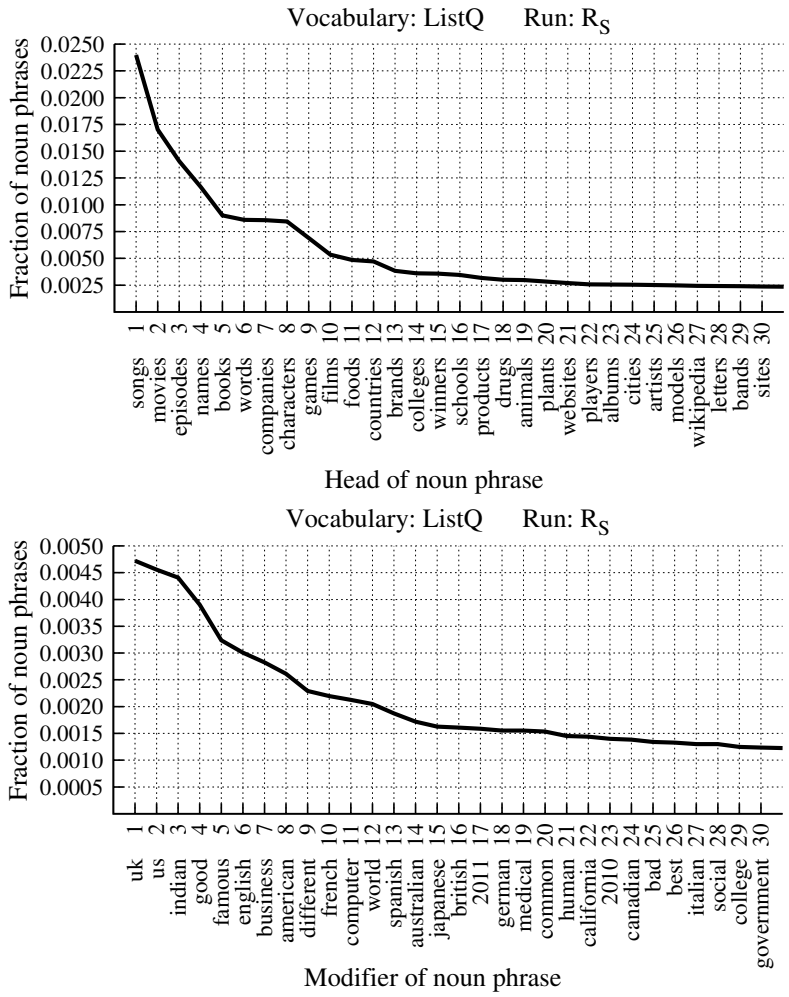

Figure 4: Most frequent heads and modifiers induced by interpretations extracted in run $\mathbf{R}_{S}$ for noun phrases from the ListQ vocabulary. Computed as the fraction of noun phrases with some extracted interpretation(s), which have a particular head (first graph) or modifier (second graph) induced by at least one of their top 10 interpretations

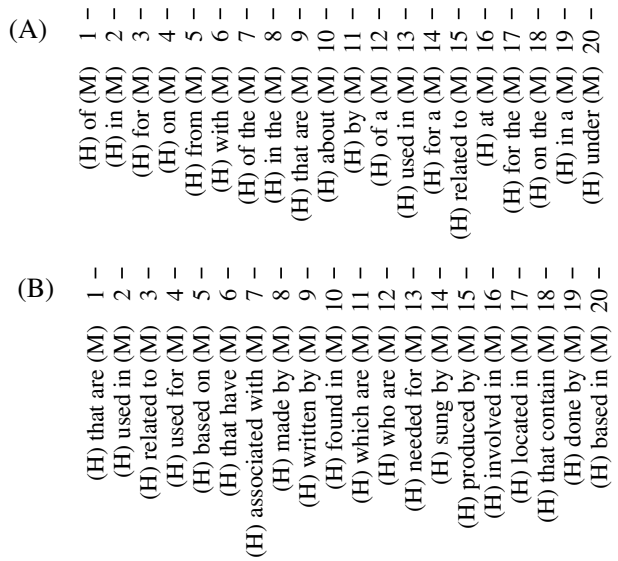

Figure 5: Most frequent interpretations extracted in run $\mathbf{R}_{S}$ for noun phrases from the ListQ vocabulary. Computed over the top 10 interpretations of noun phrases with some extracted interpretation(s). Collected interpretations are either unrestricted (A) or required (B) to include at least a token that is neither a preposition nor a determiner (second graph). (H) and (M) stand for portions of an interpretation that correspond to the head and modifier of the interpreted noun phrase

form in the interpretations. For illustration, the adj-noun variant ethiopia of the modifier ethiopian leads to the extraction of the in- 


\begin{tabular}{|c|c|c|c|c|c|}
\hline \multirow[t]{2}{*}{ Vocab } & \multirow[t]{2}{*}{ Run } & \multicolumn{4}{|c|}{ Variant Type } \\
\hline & & orig-phrase & adj-noun & dist-sim & wiki-templ \\
\hline \multicolumn{6}{|c|}{ Interpretations produced by variant type (not exclusive): } \\
\hline \multirow[t]{2}{*}{ ListQ } & $\mathrm{R}_{Q}$ & 0.563 & 0.069 & 0.642 & 0.017 \\
\hline & $\mathrm{R}_{S}$ & 0.543 & 0.069 & 0.680 & 0.019 \\
\hline \multirow[t]{2}{*}{ IsA } & $\mathrm{R}_{Q}$ & 0.526 & 0.087 & 0.595 & 0.004 \\
\hline & $\mathrm{R}_{S}$ & 0.475 & 0.085 & 0.670 & 0.005 \\
\hline \multirow[t]{2}{*}{ WikiC } & $\mathrm{R}_{Q}$ & 0.373 & 0.074 & 0.672 & 0.423 \\
\hline & $\mathbf{R}_{S}$ & 0.360 & 0.073 & 0.689 & 0.449 \\
\hline \multicolumn{6}{|c|}{ Interpretations produced only by variant type (exclusive): } \\
\hline \multirow[t]{2}{*}{ ListQ } & $\mathrm{R}_{Q}$ & 0.308 & 0.044 & 0.370 & 0.003 \\
\hline & $\mathrm{R}_{S}$ & 0.270 & 0.043 & 0.389 & 0.004 \\
\hline \multirow[t]{2}{*}{ IsA } & $\mathrm{R}_{Q}$ & 0.343 & 0.058 & 0.397 & 0.001 \\
\hline & $\mathrm{R}_{S}$ & 0.273 & 0.053 & 0.450 & 0.001 \\
\hline \multirow[t]{2}{*}{ WikiC } & $\mathrm{R}_{Q}$ & 0.128 & 0.044 & 0.284 & 0.137 \\
\hline & $\mathbf{R}_{S}$ & 0.106 & 0.042 & 0.281 & 0.144 \\
\hline
\end{tabular}

Table 6: Impact of various types of variants of modifiers, on the coverage of noun phrase interpretations. Computed as the fraction of the top 10 extracted interpretations produced by a particular variant type, and possibly by other variant types (upper portion); or produced only by a particular variant type, and by no other variant types (lower portion) (Vocab=vocabulary of noun phrases)

terpretation "runners from ethiopia" for the noun phrase "ethiopian runners". Similarly, wiki-templ variants metallica and 50 cent of the modifier art garfunkel, in the context " $X$ albums", allow for the extraction of the interpretation "albums sold by art garfunkel" for the noun phrase "art garfunkel albums", via the interpretations "albums sold by metallica" and "albums sold by 50 cent".

Table 6 quantifies the impact of various types of variants, on the coverage of noun phrase interpretations. The scores provided for each variant type correspond to either non-exclusive (upper portion of the table) or exclusive (lower portion) contribution of that variant type towards some extracted interpretations. In other words, in the lower portion, the scores capture the fraction of the top 10 interpretations that are produced only by that particular variant type. The results illustrate four phenomena. First, all variant types contribute to increasing coverage, relative to using only orig-phrase variants. Second, dist-sim variants have a particularly strong impact. Third, wiki-templ variants have a strong impact, but only when the contexts from which they were collected match the context of the noun phrase being interpreted. On the WikiC vocabulary in the lower portion of Table 6 , the scores for wiki-templ illustrate the potential that contextual variants have for extracting additional interpretations. Fourth, the relative impact of the types of variants is comparable between runs $\mathrm{R}_{Q}$ and $\mathrm{R}_{S}$.

Figure 6 quantifies the impact of various types of variants, on the coverage of noun phrase interpretations, and thus provides an alternative view to the lower portion of Table 6 . The results illustrate several phenomena. First, dist-sim variants have a particularly strong impact. Second, wiki-templ variants have a strong impact, but only when the contexts from which they were collected match the context of the noun phrase being interpreted. On the WikiC vocabulary, the scores for wiki-templ illustrate the potential of contextual variants in extracting additional interpretations. Third, beyond inherent variations, the relative impact of the types of variants is consistent between runs $\mathrm{R}_{Q}$ and $\mathrm{R}_{S}$.

Impact of Size of Textual Data: When more input queries or query sessions are available, better interpretations for more compound noun phrases may be extracted from the queries. Conversely, a reduction in the amount of textual data available in the form of queries may lead to interpretations of lower quality extracted for
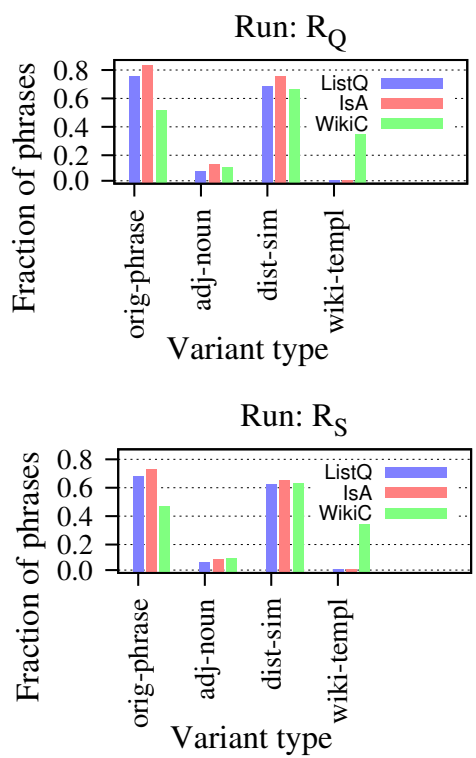

Figure 6: Impact of various types of variants of modifiers, on the coverage of noun phrase interpretations. Computed as the fraction of the noun phrases whose top 10 interpretations include some interpretation(s) produced only by a particular variant type, and by no other variant types

\begin{tabular}{|c|c|c|c|c|c|c|}
\hline \multirow{2}{*}{$\begin{array}{c}\text { Query } \\
\text { Data Size }\end{array}$} & \multirow[t]{2}{*}{$\mathrm{E}$} & \multicolumn{5}{|c|}{ Precision@N } \\
\hline & & @1 & @3 & @5 & @ 10 & @20 \\
\hline \multicolumn{7}{|c|}{ Over all evaluation phrases from ListQ } \\
\hline $1 \%$ & 42 & 0.324 & 0.278 & 0.260 & 0.242 & 0.236 \\
\hline $5 \%$ & 70 & 0.540 & 0.472 & 0.429 & 0.382 & 56 \\
\hline $10 \%$ & 76 & 0.608 & 0.534 & 0.483 & 0.433 & 0.379 \\
\hline $25 \%$ & 88 & 0.699 & 0.631 & 0.570 & 0.492 & 0.427 \\
\hline $100 \%$ & 100 & 0.886 & 0.795 & 0.726 & 0.639 & 0.549 \\
\hline \multicolumn{7}{|c|}{$\begin{array}{l}\text { Over subsets of evaluation phrases from ListQ } \\
\text { that have some some extracted interpretation(s): }\end{array}$} \\
\hline $1 \%$ & 42 & 0.648 & 0.557 & 0.520 & 0.484 & 0.473 \\
\hline $5 \%$ & 70 & 0.731 & 0.638 & 0.581 & 0.517 & 0.481 \\
\hline $10 \%$ & 76 & 0.764 & 0.671 & 0.608 & 0.544 & 0.477 \\
\hline $25 \%$ & 88 & 0.788 & 0.712 & 0.643 & 0.555 & 0.482 \\
\hline $100 \%$ & 100 & 0.886 & 0.795 & 0.726 & 0.639 & 0.549 \\
\hline
\end{tabular}

Table 7: Average precision of interpretations extracted in run $\mathbf{R}_{S}$ for noun phrases from the ListQ evaluation set, at various ranks in the ranked lists of interpretations extracted for noun phrases, when changing the size of the input query data from which interpretations are extracted. The input query data is reduced from the original data $(\mathbf{1 0 0 \%})$ to iteratively smaller data. Computed as an average over the entire set of 100 evaluation phrases from ListQ (upper portion); and as an average over (variable) subsets $\mathbf{E}$ of the 100 evaluation phrases from ListQ that have some extracted interpretation(s) (lower portion)

fewer compound noun phrases. Ablation experiments, whose results are summarized in Table 7, quantify the impact of the size of the textual data on the extracted interpretations. To this effect, the run $\mathrm{R}_{S}$ is applied to incrementally smaller input textual data. The input query session data is organized as a set of unique pairs of queries that are available as part of the same query session. From the entire query data of $100 \%$ of the query pairs, a series of iteratively smaller query data are created, in the form of random samples of $25 \%, 10 \%$ etc. of the original set of query pairs. 


\begin{tabular}{|c|c|c|c|c|c|}
\hline \multirow[t]{2}{*}{$\begin{array}{c}\text { Query } \\
\text { Data Size }\end{array}$} & \multicolumn{5}{|c|}{$\begin{array}{l}\text { Average presence of any interpretations } \\
\text { annotated as correct }\end{array}$} \\
\hline & (@) 1 & @3 & @5 & $@ 10$ & @20 \\
\hline \multicolumn{6}{|c|}{$\begin{array}{l}\text { Over subsets of evaluation phrases from ListQ } \\
\text { that have some some extracted interpretation(s): }\end{array}$} \\
\hline $1 \%$ & 0.591 & 0.659 & 0.659 & 0.659 & 0.659 \\
\hline $5 \%$ & 0.661 & 0.754 & 0.754 & 0.754 & 0.754 \\
\hline $10 \%$ & 0.714 & 0.829 & 0.843 & 0.843 & 0.843 \\
\hline $25 \%$ & 0.705 & 0.833 & 0.833 & 0.846 & 0.846 \\
\hline $100 \%$ & 0.852 & 0.909 & 0.920 & 0.932 & 0.943 \\
\hline
\end{tabular}

Table 8: Average of scores indicating the presence or absence of any interpretations annotated as correct (i.e., correct generic or correct specific). Computed for run $\mathbf{R}_{S}$ over the ListQ evaluation set, when changing the size of the input query data from which interpretations are extracted. The input query data is reduced from the original data $(100 \%)$ to iteratively smaller data. Computed over interpretations extracted up to various ranks in the ranked lists of extracted interpretations, as an average over (variable) subsets of the $\mathbf{1 0 0}$ evaluation phrases from ListQ that have some extracted interpretation(s)

As expected, there is a clear impact of the size of the input textual data, on interpretations extracted in run $\mathrm{R}_{S}$ over the ListQ evaluation set. The second column in Table 7 measures coverage. More precisely, it shows the number of evaluation phrases, out of the 100 evaluation phrases available in the ListQ evaluation set, for which some interpretations are extracted from query data of a particular size. When extracting from a quarter or a tenth of the original query data, some interpretations are extracted for 88 and 76 respectively of the 100 evaluation phrases.

The precision scores in the upper portion of Table 7 are computed as an average over all 100 evaluation phrases in ListQ. The scores quantify how precision increases, when the size of the input query data increases. But the scores are affected by both precision and coverage, rather than just precision. Indeed, when less input data is used, some of the decrease in scores can be attributed to the lack of any interpretations being extracted, for some of the evaluation phrases. Such evaluation phrases are effectively penalized, as they contribute zeros to the averages computed in the upper portion of the table. In contrast, no penalty is given to such evaluation phrases in the lower portion of Table 7. For a given input query data, the scores in the lower portion are computed over the subset of evaluation phrases with some extracted interpretations. Therefore, the scores in the lower portion of the table are a better indicator of the effect of a reduction in the size of the query data, on the quality of extracted ranked lists of interpretations. As the size of the textual data increases, the accuracy of the ranked lists of extracted interpretations also increases. Table 8 illustrates the same phenomenon, when considering the presence of any relevant interpretation, among the interpretations extracted up to a particular rank. As the size of the textual data increases, the interpretations extracted up to a particular rank include a relevant interpretation for more of the evaluation phrases, from among the subsets of evaluation phrases from ListQ for which run $\mathrm{R}_{S}$ extracts some interpretations for a given data size.

Interpretations from Queries and Sessions: An additional, hybrid run $\mathbf{R}_{H}$ merges the ranked candidate interpretations produced by $\mathrm{R}_{S}$ and $\mathrm{R}_{Q}$. More precisely, a ranked list of candidate interpretations in $\mathrm{R}_{H}$ is the concatenation of the ranked list from $\mathrm{R}_{S}$, if any, followed by the ranked list from $\mathrm{R}_{Q}$. Only the earliest occurrence of any duplicate interpretation is retained in the merged list. Thus, run $\mathrm{R}_{H}$ re-ranks interpretations that are extracted from query

\begin{tabular}{|r||r|r|r||r|r|r|}
\hline \multicolumn{1}{|c||}{ Vocab } & \multicolumn{5}{c|}{ Noun Phrases Have Interpretation(s)? } \\
\cline { 2 - 7 } & \multicolumn{4}{c|}{ Yes } & \multicolumn{4}{|c|}{ No } \\
\cline { 2 - 7 } & Count & \multicolumn{2}{c|}{ Query Freq } & Count & Query Freq \\
\cline { 2 - 7 } & $\mathrm{I}$ & $\mathrm{A}_{I}$ & \multicolumn{1}{c|}{$\mathrm{M}_{I}$} & $\neg$ & $\mathrm{A}_{\neg I}$ & $\mathrm{M}_{\neg I}$ \\
\hline \hline ListQ & 243,662 & $4.3 \times \mathrm{x}_{A}$ & $4.3 \times \mathrm{x}_{M}$ & 33,657 & $\mathrm{x}_{A}$ & $\mathrm{x}_{M}$ \\
\hline IsA & 171,938 & $8.0 \times \mathrm{y}_{A}$ & $11.5 \times \mathrm{y}_{M}$ & 12,655 & $\mathrm{y}_{A}$ & $\mathrm{y}_{M}$ \\
\hline WikiC & 39,432 & $3.6 \times \mathrm{z}_{A}$ & $8.4 \times \mathrm{z}_{A}$ & 1,172 & $\mathrm{z}_{A}$ & $\mathrm{z}_{M}$ \\
\hline
\end{tabular}

Table 9: Correlation between coverage of run $\mathbf{R}_{S}$, measured as the presence of some extracted interpretation(s) for a noun phrase, on one hand; and frequency of the noun phrase as a query, on the other hand (Vocab=vocabulary; $I=$ subset of noun phrases that are queries and have some extracted interpretation(s); $\neg I=$ subset of noun phrases that are queries and do not have any extracted interpretation(s); $A=$ =average query frequency of noun phrases as queries; $M=$ median query frequency of noun phrases as queries)

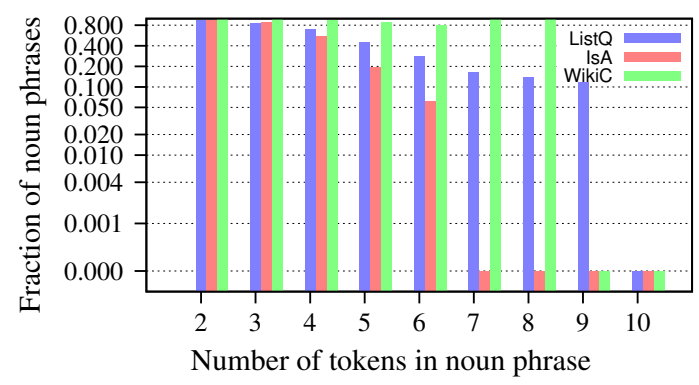

Figure 7: Ability of run $\mathbf{R}_{S}$ to extract interpretations for noun phrases, as a function of the length of (i.e., number of tokens in) noun phrases. Computed as the fraction of noun phrases from an input vocabulary with a particular number of tokens, for which there are some extracted interpretation(s)

sessions ahead of interpretations extracted only from independent queries, but otherwise keeps all interpretations extracted from independent queries as well. The precision at rank 1 is the same for $\mathrm{R}_{H}$ as for $\mathbf{R}_{S}$, over each evaluation set and also over their union. At other ranks, $\mathrm{R}_{H}$ preserves the advantage (or disadvantage) in accuracy that $\mathrm{R}_{S}$ has over $\mathrm{R}_{Q}$, but to a lesser degree. For example, when compared to earlier results in Table 3, the precision scores at ranks 5 and 20 for run $\mathrm{R}_{H}$ are 0.707 and 0.502 over the ListQ set, 0.619 and 0.396 over IsA, and 0.640 and 0.410 over WikiC.

Discussion: Independently of the source of queries (e.g., independent queries or query sessions) from which interpretations are extracted, a noun phrase is intuitively more difficult to interpret if it is relatively more rare or more complex (i.e., longer). Additional experiments quantify the effect in run $\mathrm{R}_{S}$, by measuring the correlation between the presence of some extracted interpretations for an input noun phrase, on one hand; and the frequency of the noun phrase as a query (in Table 9), on the other hand. In Table 9, the effect is visible in that query frequency is higher for noun phrases with some extracted interpretations (in the left portion of the table) vs. noun phrases with none (in the right portion). For example, for the ListQ vocabulary, the average query frequency is 4.3 higher for noun phrases with some extracted interpretations. Similarly, in Figure 7, a larger fraction of the input noun phrases with a particular number of tokens have some extracted interpretations, when the number of tokens is lower rather than higher. The effect is somewhat less pronounced for, but still applicable to, the WikiC vocabulary. That a larger fraction of the longer noun phrases can 
be interpreted in the WikiC vocabulary is attributed to the role of wiki-templ variants in extracting interpretations that would otherwise not be available.

\section{RELATED WORK}

A large body of work addresses the more general task of opendomain information extraction from text [2, 6, 38, 11, 23, 17, 41]. Extracted relations are tuples of an argument (yellow submarine), a text fragment acting as the lexicalized relation (composed by), and another argument (beatles) (cf. [11, 22]). A particular type of extracted relations are IsA relations, linking instances (yellow submarine) with their class labels ("beatles songs") $[2,8,37,20$, 7, 39, 32, 13].

Interpretations extracted from queries act as a potential bridge between facts, on one hand, and class labels, on the other hand, available for instances. The former might be inferred from the latter and vice versa. There are two previous studies that are relevant to the task of extracting facts from existing noun phrases. First, [40] extract facts for attributes of instances, without requiring the presence of the verbal predicates usually employed [11] in opendomain information extraction. Second, in [26], relations encoded implicitly within Wikipedia categories are converted into explicit relations. As an example, the explicit relation <deconstructing harry, directed, woody allen $>$ is obtained from the fact that deconstructing harry is listed under "movies directed by woody allen" in Wikipedia. The method in [26] relies on massive, manuallycompiled knowledge, and does not attempt to interpret compound noun phrases.

Relevant interpretations ("songs composed by beatles") represent alternative class labels of instances (yellow submarine) that may be originally associated with the class labels being interpreted ("beatles songs"). They may be beneficial in typed search [9] or entity search $[1,32]$. In that case, class labels submitted as queries may be answered by instances available for the class labels.

In previous work on the acquisition of paraphrases of compound noun phrases, most methods $[19,36]$ operate over documents, and may rely on text analysis tools including syntactic parsing [25]. In contrast to [28], the experiments reported here extract interpretations from query sessions, and show that the interpretations are more accurate than from independent queries. The interpretations are further related to paraphrase acquisition in that, similarly to output of other open-domain information extraction methods, they would benefit from grouping together extractions that are lexically distinct but semantically equivalent.

Queries are used as a textual data source in other tasks in opendomain information extraction [18, 31, 14]. In particular, query sessions are shown to be useful in paraphrase acquisition [42], ranking class labels extracted for instances [27] or instances extracted for class labels [3] and the disambiguation of instances mentioned in queries relative to entries in external knowledge repositories [30, 4].

\section{CONCLUSION}

The experiments described in this paper quantify the extent to which interpretations better explain the roles that modifiers play within longer noun phrases, when the interpretations are extracted from query sessions rather than independent queries.

Future work explores the role of human-curated resources such as dictionaries and encyclopedias, in guiding the acquisition of interpretations from documents and queries; and alternatives for combining interpretations from queries and from query sessions.

\section{REFERENCES}

[1] K. Balog, M. Bron, and M. de Rijke. Category-based query modeling for entity search. In Proceedings of the $32 n d$ European Conference on Information Retrieval (ECIR-10), pages 319-331, Milton Keynes, United Kingdom, 2010.

[2] M. Banko, M. J. Cafarella, S. Soderland, M. Broadhead, and O. Etzioni. Open information extraction from the Web. In Proceedings of the 20th International Joint Conference on Artificial Intelligence (IJCAI-07), pages 2670-2676, Hyderabad, India, 2007.

[3] B. Billerbeck, G. Demartini, C. Firan, T. Iofciu, and R. Krestel. Ranking entities using Web search query logs. In Proceedings of the 14th European Conference on Research and Advanced Technology for Digital Libraries (ECDL-10), pages 273-281, Glasgow, Scotland, 2010.

[4] R. Blanco, G. Ottaviano, and E. Meij. Fast and space-efficient entity linking in queries. In Proceedings of the 8th ACM Conference on Web Search and Data Mining (WSDM-15), pages 179-188, Shanghai, China, 2015.

[5] T. Brants. TnT - a statistical part of speech tagger. In Proceedings of the 6th Conference on Applied Natural Language Processing (ANLP-O0), pages 224-231, Seattle, Washington, 2000.

[6] M. Cafarella, A. Halevy, D. Wang, E. Wu, and Y. Zhang. WebTables: Exploring the power of tables on the Web. In Proceedings of the 34th Conference on Very Large Data Bases (VLDB-08), pages 538-549, Auckland, New Zealand, 2008.

[7] B. Dalvi, W. Cohen, and J. Callan. Websets: Extracting sets of entities from the Web using unsupervised information extraction. In Proceedings of the 5th ACM Conference on Web Search and Data Mining (WSDM-12), pages 243-252, Seattle, Washington, 2012.

[8] D. Davidov and A. Rappoport. Enhancement of lexical concepts using cross-lingual Web mining. In Proceedings of the 2009 Conference on Empirical Methods in Natural Language Processing (EMNLP-09), pages 852-861, Singapore, 2009.

[9] G. Demartini, T. Iofciu, and A. de Vries. Overview of the INEX 2009 Entity Ranking track. In INitiative for the Evaluation of XML Retrieval Workshop, pages 254-264, Brisbane, Australia, 2009.

[10] P. Downing. On the creation and use of English compound nouns. Language, 53:810-842, 1977.

[11] A. Fader, S. Soderland, and O. Etzioni. Identifying relations for open information extraction. In Proceedings of the 2011 Conference on Empirical Methods in Natural Language Processing (EMNLP-11), pages 1535-1545, Edinburgh, Scotland, 2011.

[12] C. Fellbaum, editor. WordNet: An Electronic Lexical Database and Some of its Applications. MIT Press, 1998.

[13] T. Flati, D. Vannella, T. Pasini, and R. Navigli. Two is bigger (and better) than one: the Wikipedia Bitaxonomy project. In Proceedings of the 52nd Annual Meeting of the Association for Computational Linguistics (ACL-14), pages 945-955, Baltimore, Maryland, 2014.

[14] M. Gamon, T. Yano, X. Song, J. Apacible, and P. Pantel. Identifying salient entities in web pages. In Proceedings of the 22nd International Conference on Information and Knowledge Management (CIKM-13), pages 2375-2380, Burlingame, California, 2013.

[15] M. Hearst. Automatic acquisition of hyponyms from large 
text corpora. In Proceedings of the 14th International Conference on Computational Linguistics (COLING-92), pages 539-545, Nantes, France, 1992.

[16] I. Hendrickx, Z. Kozareva, P. Nakov, D. Ó Séaghdha, S. Szpakowicz, and T. Veale. SemEval-2013 task 4: Free paraphrases of noun compounds. In Proceedings of the 7th International Workshop on Semantic Evaluation (SemEval-13), pages 138-143, Atlanta, Georgia, 2013.

[17] J. Hoffart, F. Suchanek, K. Berberich, and G. Weikum. YAGO2: a spatially and temporally enhanced knowledge base from Wikipedia. Artificial Intelligence Journal. Special Issue on Artificial Intelligence, Wikipedia and Semi-Structured Resources, 194:28-61, 2013.

[18] A. Jain and M. Pennacchiotti. Open entity extraction from Web search query logs. In Proceedings of the 23rd International Conference on Computational Linguistics (COLING-10), pages 510-518, Beijing, China, 2010.

[19] N. Kim and P. Nakov. Large-scale noun compound interpretation using bootstrapping and the Web as a corpus. In Proceedings of the 2011 Conference on Empirical Methods in Natural Language Processing (EMNLP-11), pages 648-658, Edinburgh, Scotland, 2011.

[20] Z. Kozareva, K. Voevodski, and S. Teng. Class label enhancement via related instances. In Proceedings of the 2011 Conference on Empirical Methods in Natural Language Processing (EMNLP-11), pages 118-128, Edinburgh, Scotland, 2011.

[21] D. Lin and X. Wu. Phrase clustering for discriminative learning. In Proceedings of the 47th Annual Meeting of the Association for Computational Linguistics (ACL-IJCNLP-09), pages 1030-1038, Singapore, 2009.

[22] Mausam, M. Schmitz, S. Soderland, R. Bart, and O. Etzioni. Open language learning for information extraction. In Proceedings of the 2012 Joint Conference on Empirical Methods in Natural Language Processing and Computational Natural Language Learning (EMNLP-CoNLL-12), pages 523-534, Jeju Island, Korea, 2012.

[23] F. Mesquita, J. Schmidek, and D. Barbosa. Effectiveness and efficiency of open relation extraction. In Proceedings of the 2013 Conference on Empirical Methods in Natural Language Processing (EMNLP-13), pages 447-457, Seattle, Washington, 2013.

[24] J. Mitchell and M. Lapata. Composition in distributional models of semantics. Cognitive Science, 34(8):1388-1429, 2010.

[25] P. Nakov and M. Hearst. Semantic interpretation of noun compounds using verbal and other paraphrases. ACM Transactions on Speech and Language Processing, 10(3):1-51, 2013.

[26] V. Nastase and M. Strube. Decoding Wikipedia categories for knowledge acquisition. In Proceedings of the $23 \mathrm{rd}$ National Conference on Artificial Intelligence (AAAI-08), pages 1219-1224, Chicago, Illinois, 2008.

[27] M. Paşca. Ranking class labels using query sessions. In Proceedings of the 49th Annual Meeting of the Association for Computational Linguistics (ACL-11), pages 1607-1615, Portland, Oregon, 2011.

[28] M. Paşca. Interpreting compound noun phrases using Web search queries. In Proceedings of the 2015 Conference of the North American Association for Computational Linguistics (NAACL-HLT-15), Denver, Colorado, 2015.
[29] P. Pantel, E. Crestan, A. Borkovsky, A. Popescu, and V. Vyas. Web-scale distributional similarity and entity set expansion. In Proceedings of the 2009 Conference on Empirical Methods in Natural Language Processing (EMNLP-09), pages 938-947, Singapore, 2009.

[30] P. Pantel and A. Fuxman. Jigs and lures: Associating web queries with structured entities. In Proceedings of the 49th Annual Meeting of the Association for Computational Linguistics (ACL-11), pages 83-92, Portland, Oregon, 2011.

[31] P. Pantel, T. Lin, and M. Gamon. Mining entity types from query logs via user intent modeling. In Proceedings of the 50th Annual Meeting of the Association for Computational Linguistics (ACL-12), pages 563-571, Jeju Island, Korea, 2012.

[32] P. Pasupat and P. Liang. Zero-shot entity extraction from Web pages. In Proceedings of the 52nd Annual Meeting of the Association for Computational Linguistics (ACL-14), pages 391-401, Baltimore, Maryland, 2014.

[33] M. Remy. Wikipedia: The free encyclopedia. Online Information Review, 26(6):434, 2002.

[34] C. Silverstein, H. Marais, M. Henzinger, and M. Moricz. Analysis of a very large Web search engine query log. SIGIR Forum, 33(1):6-12, 1999.

[35] R. Socher, J. Bauer, C. Manning, and A. Ng. Parsing with compositional vector grammars. In Proceedings of the 51st Annual Meeting of the Association for Computational Linguistics (ACL-13), pages 455-465, Sofia, Bulgaria, 2013.

[36] T. Van de Cruys, S. Afantenos, and P. Muller. MELODI: A supervised distributional approach for free paraphrasing of noun compounds. In Proceedings of the 7th International Workshop on Semantic Evaluation (SemEval-13), pages 144-147, Atlanta, Georgia, 2013.

[37] R. Wang and W. Cohen. Automatic set instance extraction using the Web. In Proceedings of the 47th Annual Meeting of the Association for Computational Linguistics (ACL-IJCNLP-09), pages 441-449, Singapore, 2009.

[38] F. Wu and D. Weld. Open information extraction using Wikipedia. In Proceedings of the 48th Annual Meeting of the Association for Computational Linguistics (ACL-10), pages 118-127, Uppsala, Sweden, 2010.

[39] W. Wu, H. Li, H. Wang, and K. Zhu. Probase: a probabilistic taxonomy for text understanding. In Proceedings of the 2012 International Conference on Management of Data (SIGMOD-12), pages 481-492, Scottsdale, Arizona, 2012.

[40] M. Yahya, S. Whang, R. Gupta, and A. Halevy. ReNoun: Fact extraction for nominal attributes. In Proceedings of the 2014 Conference on Empirical Methods in Natural Language Processing (EMNLP-14), pages 325-335, Doha, Qatar, 2014.

[41] X. Yao and B. Van Durme. Information extraction over structured data: Question Answering with Freebase. In Proceedings of the 52nd Annual Meeting of the Association for Computational Linguistics (ACL-14), pages 956-966, Baltimore, Maryland, 2014.

[42] S. Zhao, H. Wang, and T. Liu. User behaviors lend a helping hand: Learning paraphrase query patterns from search log sessions. In Proceedings of the 24th International Conference on Computational Linguistics (COLING-12), pages 3137-3152, Mumbai, India, 2012. 